\title{
Ebola Response in Liberia; Field Experiences, Challenges and Lessons Learnt during the Response, 2014-2015
}

\author{
Mutaawe Lubogo', Donewell Bangure'2, Justin Maeda ${ }^{3}$, Sasita Shabani ${ }^{3}$, \\ Theophile C. Malibiche ${ }^{3}$, Herilinda Temba ${ }^{3}$, Lucas Godbless ${ }^{3}$, Naod Bruhan ${ }^{4}$, Oketta Julius ${ }^{5}$ \\ ${ }^{1}$ Uganda Field Epidemiology Programme, Kampala, Uganda \\ ${ }^{2}$ Zimbabwe Field Epidemiology Programme, Harare, Zimbabwe \\ ${ }^{3}$ Tanzania Field Epidemiology Programme, Dar es Salam, Tanzania \\ ${ }^{4}$ Ethiopia Field Epidemiology Programme, Addis Ababa, Ethiopia \\ ${ }^{5}$ ASEOWA Head of Mission, Kampala, Uganda \\ Email: bangured@yahoo.com
}

Received 5 January 2016; accepted 22 February 2016; published 25 February 2016

Copyright (C) 2016 by authors and Scientific Research Publishing Inc.

This work is licensed under the Creative Commons Attribution International License (CC BY). http://creativecommons.org/licenses/by/4.0/

c) (i) Open Access

\section{Abstract}

The 2014-2015 Ebola Virus Disease (EVD) outbreaks which began in Guinea and later spread to Liberia, Sierra Leona and other countries have been responsible for a number of infections and deaths among the communities including health workers. The natural host of the EVD virus has not been identified even when scientific evidence points to bats as the natural reservoirs to similar strains of EVD virus. EVD male survivors have also been identified as a potential source of infection among the populations. Some of the reasons attributed to this rapid spread of EVD to other countries have been weak early warning systems to identify and notify health authorities of such diseases of high epidemic potential. Liberia has been one of the countries to be declared EVD free on two different occasions in $\mathbf{2 0 1 5}$ each with a different epicenter. Despite a number of international organizations coming together to support control efforts in Liberia, the coordination of response activities by the Ministry of Health and Social Welfare and replicated at all levels of the surveillance systems was the key in suppressing the outbreak. Adoption of the Integrated Disease Surveillance and Response (IDSR) guidelines as recommended by World Health Organization (WHO/AFRO) was a positive step towards the structuring of response activities. Capacity building of health workers in infection control and prevention and surveillance was important to improve skills of health workers to triage EVD cases as recommended by WHO. Training community health volunteers in contact tracing and active case search was important in strengthening the Early Warning Disease surveillance system. Decentralization of response activities in addition to establishing Ebola Treatment units and Community Care Centers in all counties was vital in containing the spread of infection. Even when EVD was associated to high levels of stigma, community and in- 
dividual counseling sessions led by community leaders enabled building community trust to refer cases for treatment. The EVD survivors distributed in different parts of the country are potential sources of new EVD infections. This will require strengthening early warning systems and response capacity at all levels.

\section{Keywords}

\section{Ebola, Liberia, Field Experiences, Challenges}

\section{Introduction}

Communicable disease outbreaks are associated with high morbidity and mortality in different parts of Africa despite these diseases being eradicated in other parts of the world [1]. The repeated outbreaks of such diseases in Africa are attributed to many factors including environmental degradation, population explosion, weak public health systems and encroachment of animal habitant by humans in most of these African countries [2] [3]. Because of the poor health seeking behavior among most African societies and weak early warning systems in most African countries, diseases of high epidemic potential spread and affect most people almost undetected [4]. The rampant civil wars have also contributed to weakening the health systems in addition to reducing medical access to essential medical services by the population [5].

The recent Ebola Virus Disease (EVD) outbreaks in West Africa whose epicenter was in Guinea quickly spread to Liberia and Sierra Leone due to uncontrolled movement across borders among border communities [6]. According to the latest Ebola situation report, a total of 10,675 cases have so far been infected with EVD in Liberia with 4809 EVD deaths [7].

Following the declaration of EVD as a public health event of international concern by WHO, a number of international organizations came to the region to support the government with response efforts. This joint effort led to the declaration of Liberia EVD free for the first time on the $3^{\text {rd }}$ of May 2015 [8]. Neighboring countries were meanwhile recording reducing infections in the due course. African Union Commission took immediate steps to mobilize human resources with different skills from different countries to boost response efforts in Liberia, Sierra Leone and Guinea [9]. Other countries including China and Cuba later deployed their health workers.

Following a series of epidemics that occurred in 1995 and 1996 in several countries in West and Central Africa, organizations under the leadership of WHO decided to strengthen their cooperation on Epidemic Preparedness and Response (EPR) throughout the African continent [10]. Most of the effected countries lacked essential medical supplies for effective intervention and the capacity to effectively detect and manage their epidemics was lacking. The adoption of the Integrated Disease Surveillance and Response (IDSR) guidelines by the WHO/ AFRO member countries in 1998 contributed significantly to the early detection, notification, and response to many epidemics including EVD [11]. The EVD surveillance response mechanism in Liberia was therefore modeled along these guidelines to receive information and respond appropriately in the different levels of the surveillance system. The Surveillance and Response activities were integrated in the administrative structures on the state with direct linkage form communities, health facilities, districts, counties and the national level. Each stage had prescribed roles and responsibilities with key personnel as per the IDSR guidelines. The observations noted are based on observations made by the different Field Epidemiologists under the African Union Support to Ebola Outbreak in West Africa (ASEOWA) in addition to reviewing different published literature.

\section{Coordination of the EVD Response}

When the EVD was declared by WHO as a public health disaster of international concern, different organizations came into Liberia to be part of the response activities. At national level, an Ebola task committee headed by the president and inter-ministerial committees was formed. Liberia state bi-laws were passed by the Senate aimed at controlling mass gatherings and movement of people in and outside of Liberia. Coordination of the response was conducted by the Emergency Operations Centre (EOC). The committee was responsible for advocacy and resource mobilization for EVD control. Under this committee were the technical committees that were responsible for activities like surveillance, case management, social mobilization, infection control and preven- 
tion, psychosocial support and logistical support. Such organization structures were cascaded down to the county levels with leadership from the County Superintendents and Districts Commissioners. Rapid Response teams were constituted at national, county and district level. County epidemic response activities were coordinated by the county EOCs that were linked to the national EOC in Monrovia. Such committees were supported by the international agencies across the country. These coordination committees met regularly to review the surveillance data including logistic requirements that were required to sustain the response. At the national level, this committee was chaired by the head of state and her representatives in the counties chaired similar committees at that level. Technical operation was led by Ministry of health officers at the center and county health officers in all counties.

Even when it was initially difficult to bring all partners supporting the response under one command, the establishment of county epidemic response committees was vital in allocating these partners different activities to avoid duplication of activities.

\section{Surveillance Activities in the Counties}

Scientific evidence had shown that the reproductive ratio $\left(\mathrm{R}_{\mathrm{O}}\right)$ for EVD was 2 [12], therefore this would lead to multiple infections in a short time. Given the social set up and behavior of most of African communities which encourages social mixing, caring for the sick, burial rituals. All these actions are precursors of multiple infections, In order to detect, notify and respond to cases as early as possible an early warning system was essential to respond immediately. A standard EVD case definition for epidemic setting was adopted and used throughout the epidemic [13].

Health workers in all health facilities across the country were trained on the use of standard case definition for EVD that had been modified by Liberia Ministry of Health and Social Welfare in the emergency setting. Health facilities were provided with Standard Operating Procedures (SOPs) to enable triage and isolation of suspected cases [14]. Community Health Volunteers (gCHVs) were trained to identify using the community case definition for EVD and notify cases to the toll free call center, active case finders were also trained to look for the sick people in the communities. Once a notification was received an ambulance service was sent to pick the suspected cases form the communities.

At the peak of the epidemic when the numbers of notifications were overwhelming the system, Community Care Centers were established to screen suspected cases. Those that fit the case definition or developed symptoms were immediately sent for the Ebola Treatment Unit (ETU) for further management.

Because of the limited cell phone coverage in most communities and motorized road access especially in rural Liberia, notification of cases took longest than desired. In some cases suspected cases were requested to walk to the point where the ambulance would pick them. In the capital Monrovia, identification of cases and contact tracing was hampered by the overcrowding in different communities and resistance in some of the communities to isolate the suspected cases.

Delay in isolating such cases may have also contributed to the further spread among a particular community. However this was solved by the establishment of temporary holding centers called Community Care Centers (CCCs). A system was also established to receive notifications about community deaths for safe and dignified burial. The burial teams that had been stationed in every county would then be dispatched to pick the dead, disinfect the households and also provide safe and dignified burial [15].

At the peak of the epidemic, cremation was adopted as one of the methods of disposing off the dead. This method being alien in Liberia, it caused a lot of social stigma and resentment leading to secret burials and transportation of the dead to their ancestral homes late in the night away from the cover of state security. Later during the epidemic, dead body swabbing was started to test all suspected community death for EVD.

The immigrations teams who manned check points in the country were therefore trained and equipped with tools to screen all travellers and enforce hand washing at all check points. Because of the uncontrolled movement of communities along international crossing points between countries, check points were established at all international crossing points with guinea and Sierra Leone. However, not everyone uses these points to entre another countries. The communities along these border points interact freely with each other during social functions, commerce and access to social services. These communities were therefore trained to identify the sick and link them to the Ebola task teams in border districts. Health facilities along the border points were also equipped with Infection Prevention and Control (IPC) and Personal Protective Equipment (PPE) materials to handle any reported cases before they could be picked by the ambulance services. 
Data collected at all levels of the surveillance system using both paper based and electronic systems was analyzed in different Counties and used to respond to the outbreak, A daily national situation report was shared by the Ministry of Health and Social Welfare on a daily basis. Because of the limitation in the data systems at the time, timely collection analysis and dissemination of surveillance data made response activities difficult.

\section{EVD Case Management}

Because of the many number of cases in almost all counties, Ebola treatment units (ETUs) were established in all counties. These ETUs were managed by different organizations that supported the response. It was important to manage these cases close to the source of infection to reduce potential spread. Once in ETUs, patients were screened using standard case definitions and classified as either case, probable, cases or not cases. Blood samples were taken off and sent to one of the EVD laboratories established in different counties to confirm EVD using Real Time Polymerase Chain Reaction (RT-PCR) methods as per EVD recommended testing a logarithms [16]. Following the decentralization of laboratory services and adoption of modern diagnostic equipment, the turnaround time for EVD results was reduced from 48 hours to less than 12 hours [17]. Patients who had EVD were treated using EVD treatment protocols that had been developed by a team of international experts in collaboration with the Liberian MOH [18]. Patients who did not have EVD were treated for other ailments using the Liberian national therapeutic guidelines and sent back in communities. While in the communities, these people were attached to the gCHVs for 21 day precautionary observation period. Records in various counties show that 80 new infections were recorded per 100,000 populations in the entire country between March 2014 and March 2015 with 37 survivors per 100,000 populations across the entire nation.

\section{Social Mobilization}

In order to provide clear messages about EVD for communities to be informed about the EVD signs and symptoms, sources of infection and routes of transmission, the social mobilization team at the Ministry of Health and Social Welfare with support from international agencies developed clear communication messages targeting all segments of the community. Various media of communication including community radios, print media, and social media were used to send different messages to different groups of people. The local leaders and technical teams were always provided with airtime on community radios to educate and sensitize the communities of their roles in the EVD control. In communities with no access to radio signals, posters were printed and distributed through community dialogue meetings that were organized by the task committees with different communities. In such meeting, negative messages and myths amount EVD were corrected and also restore community trust.

Community education was also adopted in all church summons where behavior change was encouraged and worshipers sat a distance away from each other.

\section{Water Sanitation and Hygiene (WASH) and Infection Prevention and Control (IPC) Activities}

In order to interrupt chains of transmission, sanitation activities were strengthened and established in all community centers, places of worship and health care facilities in accordance to WHO regulations [14]. Hand washing equipment that included buckets, chlorine, soap and thermo scans were distributed to all communities, schools and health facilities. Everyone was encouraged to regularly wash hands in an effort to protect oneself and his family. Health facilities were assessed for IPC requirements and provided according to need. The IPC materials included PPEs, thermo scans and hand washing facilities. Health workers were trained on how to use the PPEs and triage all patients that presented for treatment. In different counties, the WASH and IPC activities were supported by different partners in collaboration with respective county health teams. These partners also supported the establishment of triage facilities in all health facilities across the country.

In an effort to restore essential education services by the opening of schools, IPC assessment prior to school opening was conducted. Schools that acted as CCCs were disinfected and all schools were supported to establish Ebola tasks teams with clear roles and responsibilities. These teams were trained to ensure that all pupils were screened twice a day and ensure that hand washing facilities were available with every school having one entry point. 


\section{Psychosocial Support}

Ebola infections have been associated with many psychosocial ailments among the affected communities and survivors. Ailments range from stigmatization of the victims by the communities and also the effect of the post traumatic distress syndrome among survivors. Psychosocial management was therefore very important in building community trust to refer suspected cases and ease of integration of survivors in the communities of origin.

Because of the limited number of qualified human resource in psychosocial counseling, community leaders including religious and cultural leaders were given the responsibility of conducting community counseling sessions. Routine visits would also be made to follow up on the families of the people who died of EVD.

Communities were allowed to visit the sick relatives in the ETUs and talk to the sick relatives. Some of them would be informed on the death of their relatives and attend their burials. Photos of the deceased would then be given to the relatives as confirmation of death of their loved one.

Such a strategy contributed significantly to building community trust and demystifying the ETUs which were earlier labeled death traps because most of the people referred to these ETUs died on admission. Allowing relatives to talk to the sick while under admission was one of the ways to begin integration of the survivors back to their communities.

\section{Logistic Support}

In order to respond to any epidemic appropriately, logistical support is very critical. Logistical requirements in such setting should be aligned to the needs of a particular epidemic. It was therefore critical to establish a logistical committee as part of the rapid response teams. Logistical bases were established in all counties and distribution made to all health facilities based on need. Emergency supplies for PPEs and medical supplies were deposited at the depots in all counties and were distributed using the established supply chain management systems. Supply requirements included IPC supplies and essential medical supplies and equipment. However, EVD being a new infection in Liberia and due to absence of enabling laws to facilitate emergency procurement, supply challenges were experienced in the earlier periods of the response.

\section{Discussion}

Despite the recent EVD outbreak being the biggest EVD outbreak ever recorded anywhere in the world, decentralized response was key in reducing the spread of transmission of EVD cases. The establishment of the EOC as the coordinating center for EVD response across all levels of the response system was key in coordination of response. This was important in aligning response needs to particular county requirements.

Like in other countries with history of similar EVD outbreaks, establishment of rapid response teams in all districts with appropriate skills mix as per IDSR guidelines and community engagement was very important in early detection, notification and isolation [19] [20]. Establishing CCCs and ETUS in all counties was important in limiting the transportation of cases over long distances.

Unlike in other countries which had prior experience in handling of EVD outbreaks, Liberia had challenges in the earlier outbreaks [20] [22]. This is evidenced by the high infection rates and case fatalities among health workers [22] [23]. Training health workers on job in IPC and later in basic disease surveillance was critical in not only protecting health workers but also building capacity for any future outbreaks [24] [25]. The declaration of the EVD outbreak as a public health event of international concern was critical in creating awareness of the need for international support. It was therefore important that African union came in handy to send a work force of different health professionals to boost the response activities in different counties [26]. Other organizations followed suite by providing both human resources and logistical support to the response.

Scientific evidence has demonstrated that male survivors harbor the virus for up to 90 days [22] [27]. This therefore implies that other outbreaks are more likely to occur in communities with such survivor [23]. Even when survivors are provided with the necessary health education about safe sex and condoms provided, their use cannot be guaranteed. This therefore calls for strengthening of early warning systems and response mechanisms at all levels [28]. This will also require simulation exercises for the health worker force and Rapid Response teams to keep their response skills up to date.

Even when some species of viral hemorrhagic fevers have been demonstrated to be hosted in various mammals in the environment, more research is required to identify the natural host of the EVD virus [29] [30]. This 
will enable scientists develop appropriate strategies to protect the populations against infections. In addition further research is required to determine the efficacies of various vaccines under trials in addition to studying the natural history of the EVD virus in the human host. Because Africa has experience various outbreaks of infectious disease in the recent past attributed to partly environmental encroachment, migration due to population pressure and global warming, a continental body needs to be established as a stand by force for emergency deployment in case of any other such outbreak anywhere in Africa.

\section{Conclusion}

The response mechanism to EVD in Liberia has provided valuable lessons to all countries in the world of the importance of decentralization of response, inter-sectorial collaboration, partners support and coordination and strengthening of existing structures to avert any such outbreak. However, risk mapping for all potential sources of future outbreaks should be undertaken, epidemic response plans developed and required logistics stocked to be able to manage any future outbreaks timely to avert potential spread to a wider community.

\section{Acknowledgements}

We thank the African Union for the logistical support during the deployment, Centers for Disease Control and Prevention for the technical assistance during the deployment and Liberian Ministry of Health and Social Welfare for the good working relationship.

\section{Competing Interests}

The author declares no competing interests.

\section{Authors' Contributions}

All authors were responsible for the conception of the problem, design, collection, analysis and interpretation of data and drafting of the final article. The manuscript was read and approved by all authors.

\section{References}

[1] Kebede, S., et al. (2010) Trends of Major Disease Outbreaks in the African Region, 2003-2007. East African Journal of Public Health, 7, 20-29.

[2] Mayega, R.W., et al. (2013) A Descriptive Overview of the Burden, Distribution and Characteristics of Epidemics in Uganda. East African Journal of Public Health, 10, 397-402.

[3] Feldmeier, H., Komazawa, O. and Moji, K. (2014) Nodding Syndrome in Uganda: Field Observations, Challenges and Research Agenda. Tropical Medicine and Health, 42, 109-114. http://dx.doi.org/10.2149/tmh.2014-S15

[4] Alexander, K.A., et al. (2015) What Factors Might Have Led to the Emergence of Ebola in West Africa? PLoS Neglected Tropical Diseases, 9, e0003652. http://dx.doi.org/10.1371/journal.pntd.0003652

[5] McPake, B., et al. (2015) Ebola in the Context of Conflict Affected States and Health Systems: Case Studies of Northern Uganda and Sierra Leone. Conflict and Health, 9, 23. http://dx.doi.org/10.1186/s13031-015-0052-7

[6] WHO (2014) Ebola Virus Disease Outbreak in Guinea. Disease Outbreak News, 1, 1.

[7] WHO (2015) Ebola Situation Report. Ebola Reports, 1, 15.

[8] WHO (2015) WHO Statement. Ebola Reports, 1, 15.

[9] Commission, A. (2014) Decision on Ebola Virus Disease (EVD) Outbreak. Executive Council Sixteenth Extraordinary Session, 1, 4.

[10] WHO/AFRO (2001) Epidemic Preparedeness and Response in Africa. An Epidemiological Block Approach. Report, 1, 20.

[11] Kasolo, F., Yoti, Z., Bakyaita, N., Gaturuku, P., Katz, R., Fischer, J.E. and Perry, H.N. (2013) IDSR as a Platform for Implementing IHR in African Countries. Biosecurity and Bioterrorism: Biodefense Strategy, Practice, and Science, 11, 163-169. http://dx.doi.org/10.1089/bsp.2013.0032

[12] Van Kerkhove, M.D., Bento, A.I., Mills, H.L., Ferguson, N.M. and Donnelly, C.A. (2015) A Review of Epidemiological Parameters from Ebola Outbreaks to Inform Early Public Health Decision-Making. Scientific Data, 2, Article No.: 150019. http://dx.doi.org/10.1038/sdata.2015.19 
[13] WHO (2014) Case Definitions Recommendations of Ebola or Murburg Virus Diseases. Interim Guidelines, 1, 4.

[14] WHO (2014) Infection Prevention and Control Guidelines for Care of Patients with Suspected or Confirmed Filo Virus Haemorrhagic Fevers in Health Care Settings with Focus on Ebola. Interim Guidelines, 1, 27.

[15] WHO (2014) How to Conduct Safe and Dignified Burrial of a Patient Who Has Died from Suspected or Confirmed Ebola Virus Disease. Interim Guidelines, 1, 27.

[16] WHO (2014) Laboratory Diagnosis of Ebola Virus Disease-Interim Guidelines. Interim Guidelines, 1, 20.

[17] Nouvellet, P., Garske, T., Mills, H.L., Nedjati-Gilani, G., Hinsley, W., Blake, I.M., et al. (2015) The Role of Rapid Diagnostics in Managing Ebola Epidemics. Nature, 528, S109-S116. http://dx.doi.org/10.1038/nature16041

[18] WHO (2015) Ebola V Manual for the Care and Management of Patients in Ebola Care Units/Community Care Centres. WHO/EVD/Manuals/ECU/15.1, p. 20.

[19] Mbonye, A., Wamala, J., Winyi-Kaboyo, Tugumizemo, V., Aceng, J. and Makumbi, I. (2012) Repeated Outbreaks of Viral Hemorrhagic Fevers in Uganda. African Health Sciences, 12, 579-583.

[20] Mbonye, A.K., Wamala, J.F., Nanyunja, M., Opio, A., Makumbi, I. and Aceng, J.R. (2014) Ebola Viral Hemorrhagic Disease Outbreak in West Africa-Lessons from Uganda. African Health Sciences, 14, 495-501. http://dx.doi.org/10.4314/ahs.v14i3.1

[21] Arwady, M.A., Bawo, L., Hunter, J.C., Massaquoi, M., Matanock, A., Dahn, B., et al. (2015) Evolution of Ebola Virus Disease from Exotic Infection to Global Health Priority, Liberia, Mid-2014. Emerging Infectious Diseases, 21, 578-584. http://dx.doi.org/10.3201/eid2104.141940

[22] Tseng, C.P. and Chan, Y.J. (2015) Overview of Ebola Virus Disease in 2014. Journal of the Chinese Medical Association, 78, 51-55. http://dx.doi.org/10.1016/j.jcma.2014.11.007

[23] Petti, S., Messano, G.A., Vingolo, E.M., Marsella, L.T. and Scully, C. (2015) The Face of Ebola: Changing Frequency of Haemorrhage in the West African Compared with Eastern-Central African Outbreaks. BMC Infectious Diseases, 15, 564. http://dx.doi.org/10.1186/s12879-015-1302-4

[24] Bazeyo, W., Bagonza, J., Halage, A., Okure, G., Mugagga, M., Musoke, R., et al. (2015) Ebola a Reality of Modern Public Health; Need for Surveillance, Preparedness and Response Training for Health Workers and Other Multidisciplinary Teams: A Case for Uganda. The Pan African Medical Journal, 20, 404. http://dx.doi.org/10.11604/pamj.2015.20.404.6159

[25] Nguku, P., Mosha, F., Prentice, E., Galgalo, T., Olayinka, A. and Nsubuga, P. (2015) Field Epidemiology and Laboratory Training Programs Have Been in Africa for 10 Years, What Is Their Effect on Laboratory-Based Surveillance? Reflections from a Panel at the African Society of Laboratory Medicine December 2014 Cape Town Meeting. The Pan African Medical Journal, 20, 451. http://dx.doi.org/10.11604/pamj.2015.20.451.6787

[26] Lubogo, M., Donewell, B., Godbless, L., Shabani, S., Maeda, J., Temba, H., Malibiche, T.C. and Berhanu, N. (2015) Ebola Virus Disease Outbreak; the Role of Field Epidmiology Training Program in the Fight against the Epidemic, Liberia, 2014. The Pan African Medical Journal, 22, 5.

[27] Deen, G.F., Knust, B., Broutet, N., Sesay, F.R., Formenty, P., Ross, C., et al. (2015) Ebola RNA Persistence in Semen of Ebola Virus Disease Survivors-Preliminary Report. The New England Journal of Medicine. http://dx.doi.org/10.1056/NEJMoa1511410

[28] Nsubuga, P. (2014) The Ebola Outbreak in West Africa: A Story of Related Public Health Challenges and a Pointer to Solutions to Mitigate the Inevitable Next Outbreak. The Pan African Medical Journal, 19, 48. http://dx.doi.org/10.11604/pamj.2014.19.48.5336

[29] Jayme, S.I., Field, H.E., de Jong, C., Olival, K.J., Marsh, G., Tagtag, A.M., et al. (2015) Molecular Evidence of Ebola Reston Virus Infection in Philippine Bats. Virology Journal, 12, 107. http://dx.doi.org/10.1186/s12985-015-0331-3

[30] Anti, P., Owusu, M., Agbenyega, O., Annan, A., Badu, E.K., Nkrumah, E.E., et al. (2015) Human-Bat Interactions in Rural West Africa. Emerging Infectious Diseases, 21, 1418-1421. http://dx.doi.org/10.3201/eid2108.142015 